\title{
Health systems resilience in managing the COVID-19 pandemic: lessons from 28 countries
}

\author{
Victoria Haldane ${ }^{10}$ 1, , Chuan De Foo ${ }^{2,9}$, Salma M. Abdalla3,4,9, Anne-Sophie Jung ${ }^{5,9}$, Melisa Tan², \\ Shishi Wu${ }^{2}$, Alvin Chua ${ }^{2}$, Monica Verma ${ }^{2}$, Pami Shrestha ${ }^{2}$, Sudhvir Singh ${ }^{4,6}$, Tristana Perez ${ }^{5}$, \\ See Mieng Tan' ${ }^{2}$, Michael Bartos ${ }^{4,7}$, Shunsuke Mabuchi ${ }^{4}$, Mathias Bonk ${ }^{4,8}$, Christine McNab4, \\ George K. Werner ${ }^{4}$, Raj Panjabi ${ }^{4}$, Anders Nordström ${ }^{4}$ and Helena Legido-Quigley ${ }^{2,4,5} \bowtie$
}

\begin{abstract}
Health systems resilience is key to learning lessons from country responses to crises such as coronavirus disease 2019 (COVID-19). In this perspective, we review COVID-19 responses in 28 countries using a new health systems resilience framework. Through a combination of literature review, national government submissions and interviews with experts, we conducted a comparative analysis of national responses. We report on domains addressing governance and financing, health workforce, medical products and technologies, public health functions, health service delivery and community engagement to prevent and mitigate the spread of COVID-19. We then synthesize four salient elements that underlie highly effective national responses and offer recommendations toward strengthening health systems resilience globally.
\end{abstract}

C OVID-19 has caused an unprecedented global crisis, including millions of lives lost, public health systems in shock and economic and social disruption, disproportionately affecting the most vulnerable. As of April 2021, there are over 140 million confirmed cases and over three million COVID-19 deaths globally ${ }^{1}$. While vaccination has commenced in numerous countries, new outbreaks and variants continue to emerge. At the same time, the global distribution of vaccines is marred by challenges of equity on top of logistical complications. Millions more are therefore still at risk of dying, facing significant morbidity or losing their livelihoods given the uncertain economic outlook.

The pandemic has challenged local, national, regional and global capacities to prepare and respond. The various national strategies taken to control viral transmission are widely debated ${ }^{2,3}$. However, the relative success of these strategies depends largely on how an existing health system is organized, governed and financed across all levels in a coordinated manner ${ }^{4}$. The pandemic has exposed the limitations of many health systems, including some that have been previously classified as high performing and resilient ${ }^{5}$. A comprehensive analysis of the resilience of health systems during the pandemic can therefore pinpoint important lessons and help strengthen countries' preparedness, response and approach to future health challenges.

While resilience is a core concept in disaster risk reduction, its application to health systems is relatively new. It has been defined broadly as institutions' and health actors' capacities to prepare for, recover from and absorb shocks, while maintaining core functions and serving the ongoing and acute care needs of their communities $^{6,7}$. During a crisis, a resilient health system is able to effectively adapt in response to dynamic situations and reduce vulnerability across and beyond the system. Experience from previous epidemics, such as Ebola, severe acute respiratory syndrome (SARS) and Middle East respiratory syndrome, emphasized the links between resilience and thwarting new outbreak transmission ${ }^{8}$.
Health systems resilience literature stresses that efforts should focus not only on absorbing unforeseen shocks precipitated by emerging health needs, but also on ensuring continuity in health improvement, sustaining gains in systems functioning and fostering people centeredness, while delivering high-quality care ${ }^{9,10}$. As COVID-19 has overwhelmed health systems worldwide, debates around resilience have become more urgent, and there is a need to better understand the elements of national responses through a resilience lens ${ }^{11,12}$. Thus, in this perspective, we use an expanded health systems resilience framework centered on community engagement to examine 28 national responses to COVID-19. This analysis provides insights into the policies countries implemented and how these were implemented to tackle the pandemic.

Conceptual framework. Our conceptual framework (Fig. 1) is grounded in the World Health Organization's (WHO) health systems framework ${ }^{13}$. We develop the framework elements by adding public health functions, including testing, contact tracing, disease surveillance and non-pharmaceutical public health interventions, which often operate separately from health service delivery. Yet, they are critical both to pandemic responses and to ongoing population health. Similarly, health information systems are vital functions for both public health and health systems as, ideally, they should be integrated to capture data at individual, health system and population levels.

We centered our analysis on community engagement as core to all elements of health systems resilience (see Box 1 for more information on the analytical approach). To serve communities in a more equitable manner and promote healthy societies, resilience must be developed with these communities and according to their needs. There can be no health systems resilience without community engagement across domains ${ }^{14}$. We also acknowledge the critical role of coordination with non-health sectors as essential to providing necessary supports to address the social determinants

IInstitute of Health Policy, Management and Evaluation, University of Toronto, Toronto, Ontario, Canada. ${ }^{2}$ Saw Swee Hock School of Public Health, Singapore, Singapore. ${ }^{3}$ School of Public Health, Boston University, Boston, MA, USA. ${ }^{4}$ The Independent Panel for Pandemic Preparedness and Response Secretariat, Geneva, Switzerland. ${ }^{5}$ London School of Hygiene and Tropical Medicine, London, UK. ${ }^{6}$ Faculty of Medical and Health Sciences, University of Auckland, Auckland, New Zealand. ${ }^{7}$ School of Sociology, Australian National University, Canberra, New South Wales, Australia. ${ }^{8}$ Berlin Institute of Global Health, Berlin, Germany. ${ }^{9}$ These authors contributed equally: Victoria Haldane, Chuan De Foo, Salma M. Abdalla, Anne-Sophie Jung. 凶e-mail: ephhlq@nus.edu.sg 


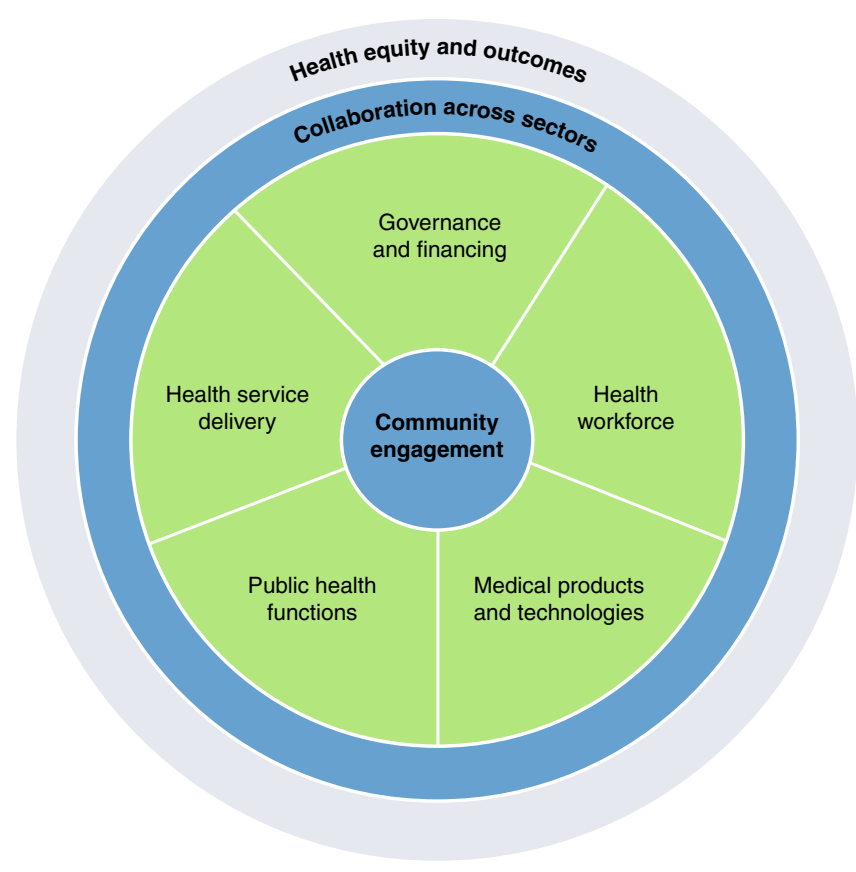

Fig. 1 | Determinants of health systems resilience framework. The scheme illustrates the components of the resilient health systems framework we developed based on the WHO's health systems building blocks framework. The five elements of resilient health systems are centered around community engagement as core to all elements of health systems resilience.

of health. Underpinning these elements are health equity and outcomes. Resilient health systems should aim to generate positive physical and mental health outcomes for all, including vulnerable and marginalized groups. In many countries, COVID-19 mortality rates have been disproportionately higher among older populations, minority ethnic groups, socioeconomically deprived populations and low-wage and migrant workers, emphasizing the interconnectedness between equity and health outcomes ${ }^{15}$.

\section{COVID-19 responses in $\mathbf{2 8}$ countries}

Using our framework, we organized our results beginning with domains often viewed as external to health, which are nevertheless central determinants of health systems resilience-governance, finance, collaboration across sectors and community engagementbefore exploring domains more closely associated with traditional views of health and health systems-health service delivery, health workforce, medical products and technologies and public health functions. We offer illustrative examples of selected countries for each domain in Tables $1-6$. We analyzed 28 countries based on a purposive selection, including positive and negative outliers in relation to reported COVID-19 deaths per capita among highly populous countries, as well as a selection of countries in the middle ground (as of 6 November 2020). Figure 2 provides an overview of countries in our review.

Governance, finance and collaboration across sectors. COVID-19 has made policymakers and the wider public acutely aware of the relationship between health systems, domestic economies and governance. Government decisions determine healthcare infrastructures, regulations and guidelines, defining access to medication and treatment, the provision of health coverage and the financing of these. Government responses to COVID-19, in the immediate term, have meant the difference between lockdown or business as usual, and have eroded or increased public trust. In the longer term, they have shaped national choices regarding private healthcare or

\section{Box 1 | Analytical approach}

The analysis presented in this review is based on a purposive selection of countries including positive and negative outliers in relation to reported COVID-19 deaths per capita among highly populous countries, as well as a selection of countries in the middle ground from different regions and with widely varying health systems and economic status. Figure 2 provides an overview of countries in our review. Countries were selected according to the recorded number of deaths attributed to COVID-19 per 100,000 inhabitants on 6 November 2020. Given the dynamic and continuously changing situation, the ranking of positive and negative outliers in terms of death per capita may have changed during the pandemic. The analysis is thus limited to this specific time period.

Five complementary methods were adopted to analyze national responses to COVID-19:

1. Literature review: using standardized methods, we identified peer-reviewed papers and public reports that examined national and subnational policy responses and extracted data for each country on five dimensions comprising 62 items. The dimensions and items were identified through a review of 14 existent frameworks.

2. Semi-structured interviews and national government submissions: to supplement the literature review, we asked the selected countries and country experts a set of questions related to the measures implemented to contain COVID-19 in their respective countries. A total of 45 interviews and written submissions were provided. Semi-structured interviews with COVID-19 national experts at the policy, operational and academic levels were recorded and transcribed in full. All interviews were coded through an inductive approach and thematic analysis, using QSR NVivo 12 software, drawing on techniques of the constant comparison method.

3. In-depth case studies of 6 countries to validate the data from the 28 countries.

4. Expert validation of country-specific data with country experts. Experts in countries were provided with the specific data for each of their respective countries to validate the data.

5. Expert validation through roundtable discussions with country experts. A total of 35 national and international experts in COVID-19 policies participated in two roundtable discussions.

universal health coverage (UHC) and strengthened or impoverished social safety nets that underpin health and well-being.

Overall, COVID-19 responses saw health policy moving beyond the remit of Ministries of Health and in doing so, draw on expertise from other ministries, particularly during the early response. Countries took whole-of-government approaches to strengthen health systems in response to COVID-19, particularly those with experience of other health-related disasters, such as Ebola. These decisions were, in most countries, made by translating evidence-based research into policies that preserve health system capacity, while protecting both public health and livelihoods. As such, most countries established temporary COVID-19 advisory groups to inform government decisions. However, in the majority of countries, the views represented on these committees were largely biomedical. More information on national responses from a governance and leadership perspective is available (V.H., A.-S.J., R. Neill, S.W. and M. Jamieson, unpublished data).

The COVID-19 response requires testing, treatment and vaccines to be financed with either a portion or all of these costs 

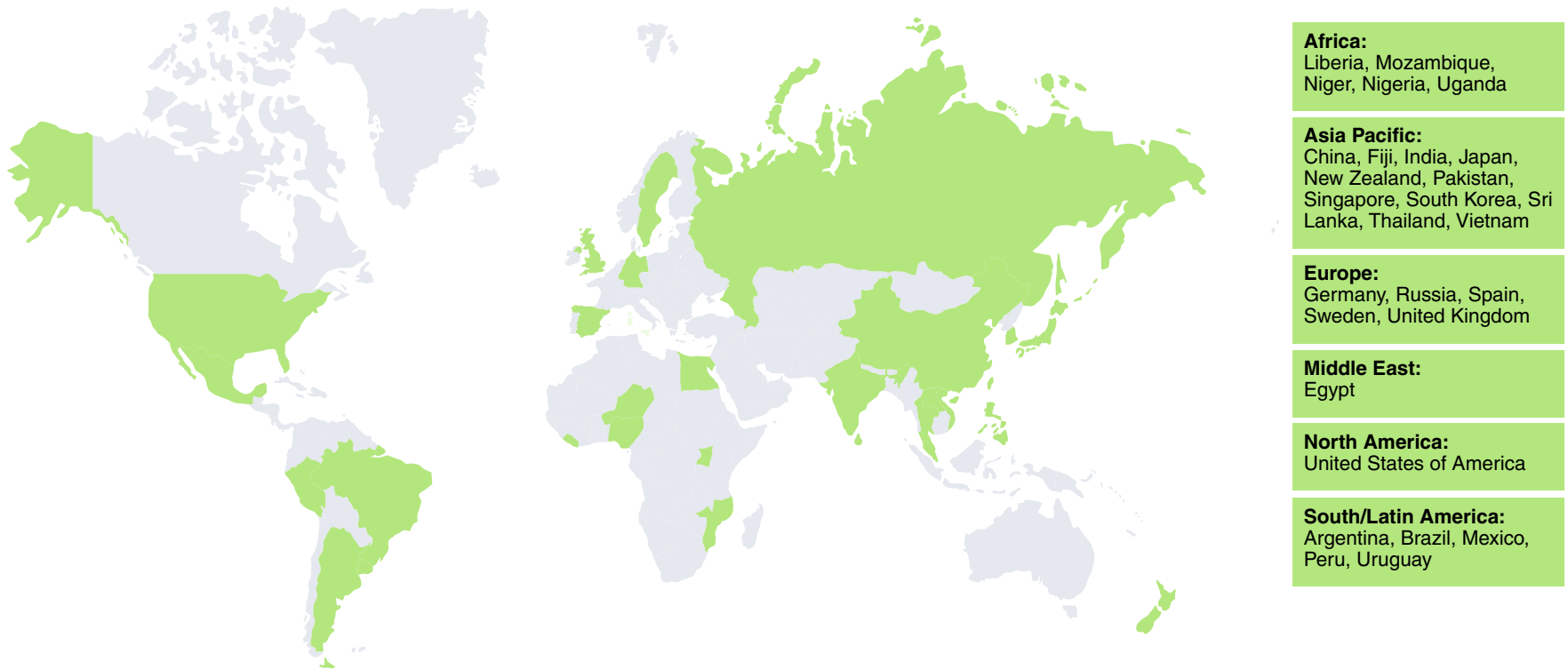

Fig. 2 | Countries included in the review. The map presents an overview of countries selected according to the recorded number of deaths attributed to COVID-19 per 100,000 inhabitants on 6 November 2020. Countries are listed according to region and in alphabetical order.

coming from public funds, and depends on healthcare infrastructures, workforce and supplies to provide much-needed surge capacity within health systems. Additionally, fiscal support measures, including relief packages aiming at helping businesses stay viable, protect jobs or provide financial aid to low-income households and the unemployed have been taken by many countries. These policies support people to adhere to public health guidance, with the aim of preventing infections, in turn mitigating the strain on the health system resulting from the need to deliver high-intensity COVID-19 care. To further enhance the reach of health services, many countries reviewed took specific actions to mitigate potential financial and physical barriers to care, such as covering part or all of the costs of COVID-19 care, and funding the establishment of testing and treatment centers in communities ${ }^{16}$. Importantly, the outcomes resulting from financial expenditures on health and well-being are only as good as the structures that support them. Thus, they require governance expertise across levels, sectors and domains and depend on a system's ability to reach (vulnerable) populations.

Community engagement. Deep engagement with local communities is central to resilient health systems as a way to inform service delivery, decision-making and governance and to meet the needs of communities before, during and after crises. Community engagement strategies, such as building partnerships with local leaders and working alongside community members to tailor messages and campaigns are crucial during public health emergencies ${ }^{17}$. The range of non-pharmaceutical public health interventions employed in response to COVID-19, such as mask wearing and social distancing, rely on shared values and a sense of social responsibility within communities to break chains of viral transmission ${ }^{18}$.

Several countries reviewed engaged networks of community health workers (CHWs) to encourage active community participation in COVID-19 responses. Their roles range from creating awareness through door-to-door visits, supporting contact tracing efforts, maintaining essential health services, providing necessary medication to patients without COVID-19, surveillance or monitoring adherence to quarantine measures and assessing mental well-being ${ }^{19}$. They are also key to identifying and referring patients who face barriers to accessing healthcare services. For example, Thailand deployed over 1 million $\mathrm{CHW}$ to disseminate and amplify messages widely in communities. Singapore deployed volunteers to educate seniors and help distribute daily necessities. Liberia further empowered community leaders by providing orientation on COVID-19 epidemiology to support containment efforts. However, many of these efforts depended on volunteers.

During early response efforts, a few countries conducted surveys to understand public sentiment regarding evolving measures. Japan conducted a survey in April 2020 to understand compliance with social distancing measures, using the results to inform response strategies. Governments also started multilingual hotlines to ensure comprehensive access to COVID-19 information. Other countries used social media platforms to engage communities. For example, the \#TakeResponsibility campaign in Nigeria called on citizens to join forces and be proactive in taking greater individual and collective responsibility in controlling the spread of COVID- $19^{20}$.

Health service delivery. Health systems globally have employed three common approaches to rapidly scale up health system infrastructure, namely by constructing new treatment facilities, converting public venues and reconfiguring existing medical facilities to provide care for patients with COVID-19. Thus, some of the health systems reviewed invested significant resources into rapidly creating dedicated field facilities. For example, in early 2020, China established two specialty field hospitals in under 2 weeks ${ }^{21}$. Where field hospitals were set up to house patients with COVID-19, countries often drew on their armed forces and military field hospital models, or adapted existing large public facilities. However, most health systems relied on a less resource-intensive approach that modified traditional healthcare facilities into dedicated COVID-19 care centers $^{22}$. Other health systems relied on home care for patients with mild to moderate COVID-19, with facilities available if patients were unable to safely self-isolate within their homes. Additionally, the majority of reviewed countries canceled elective surgeries in an effort to ensure system capacity for COVID-19 care.

In many health systems, primary-care providers are the frontline of the health system providing continuous, coordinated and people-centered care. Primary care is an important point of COVID-19 triage, as well as the point at which most routine and acute care services are provided within communities ${ }^{23}$. In many of the countries reviewed, primary-care providers rapidly adopted and scaled up digital technology or telehealth services to provide ongoing and acute care while also triaging and referring persons with 


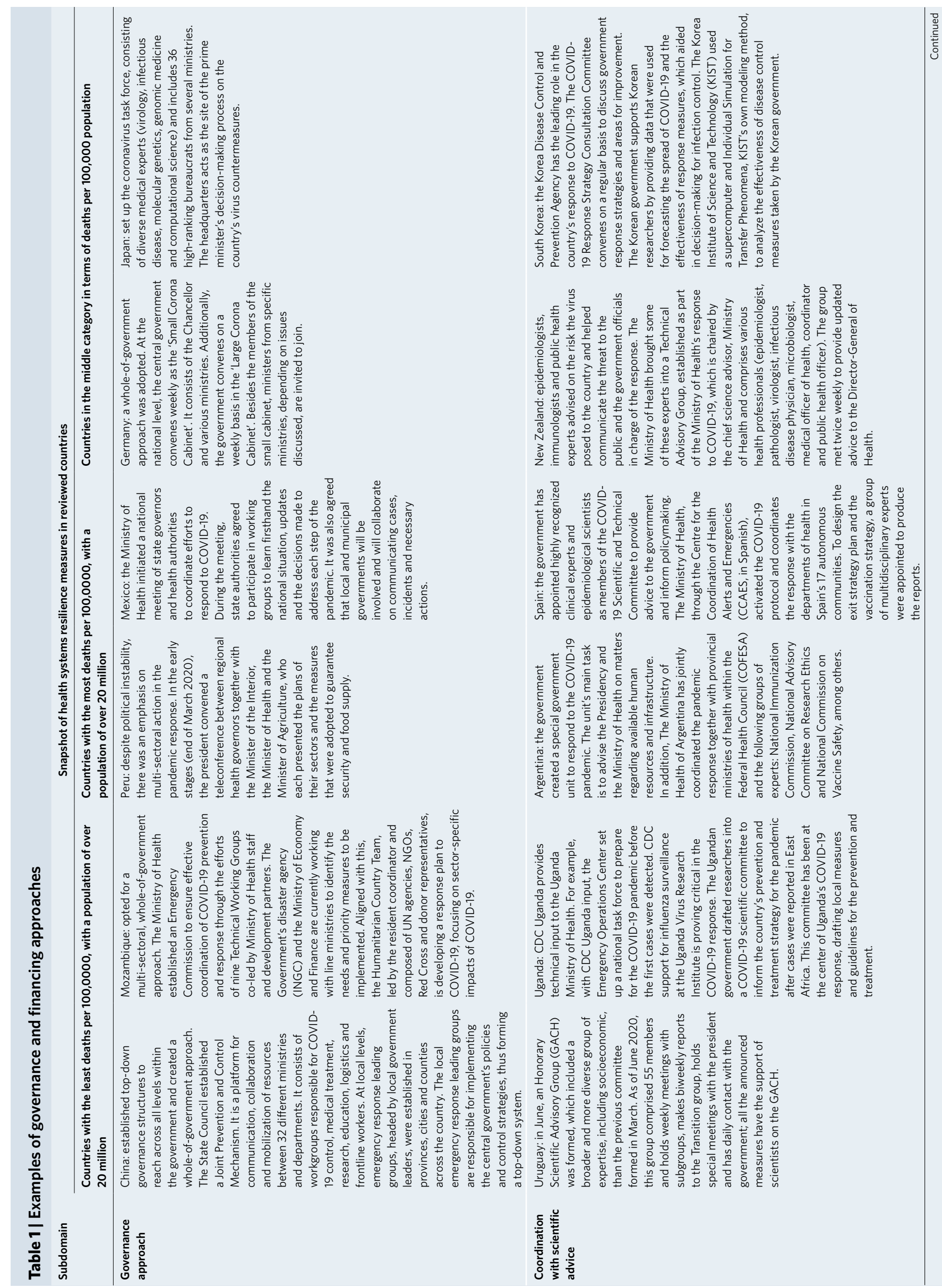




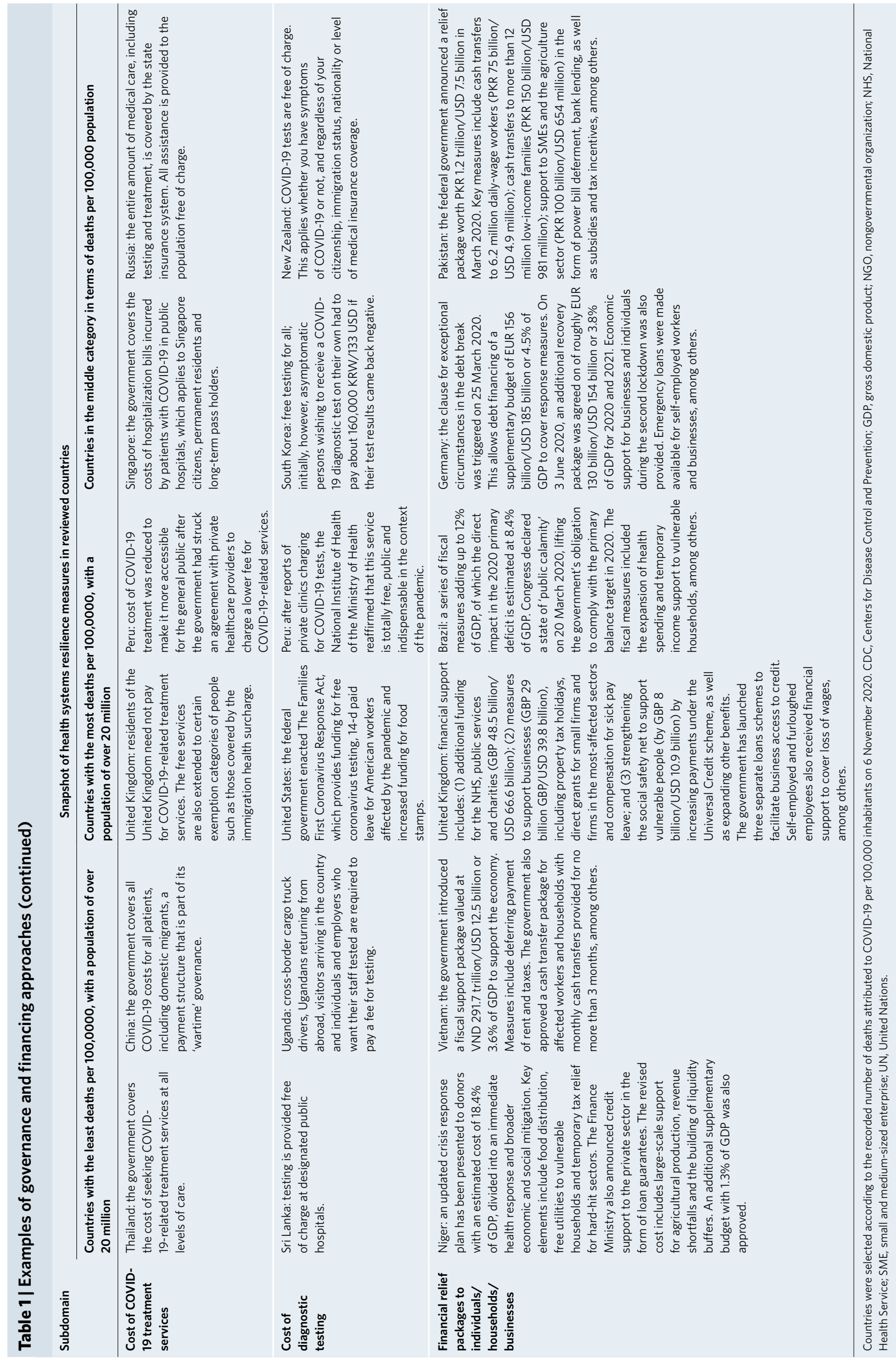




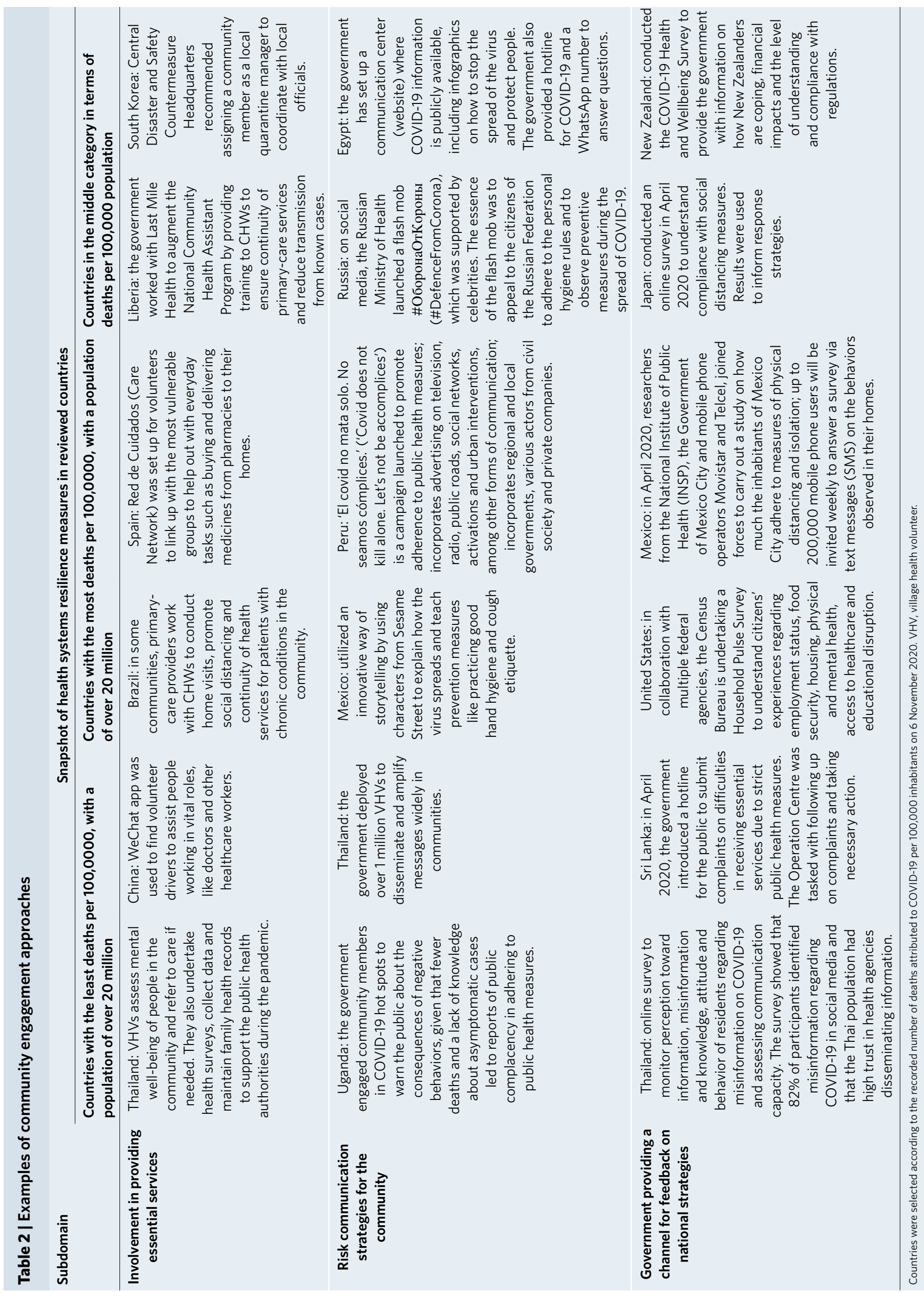




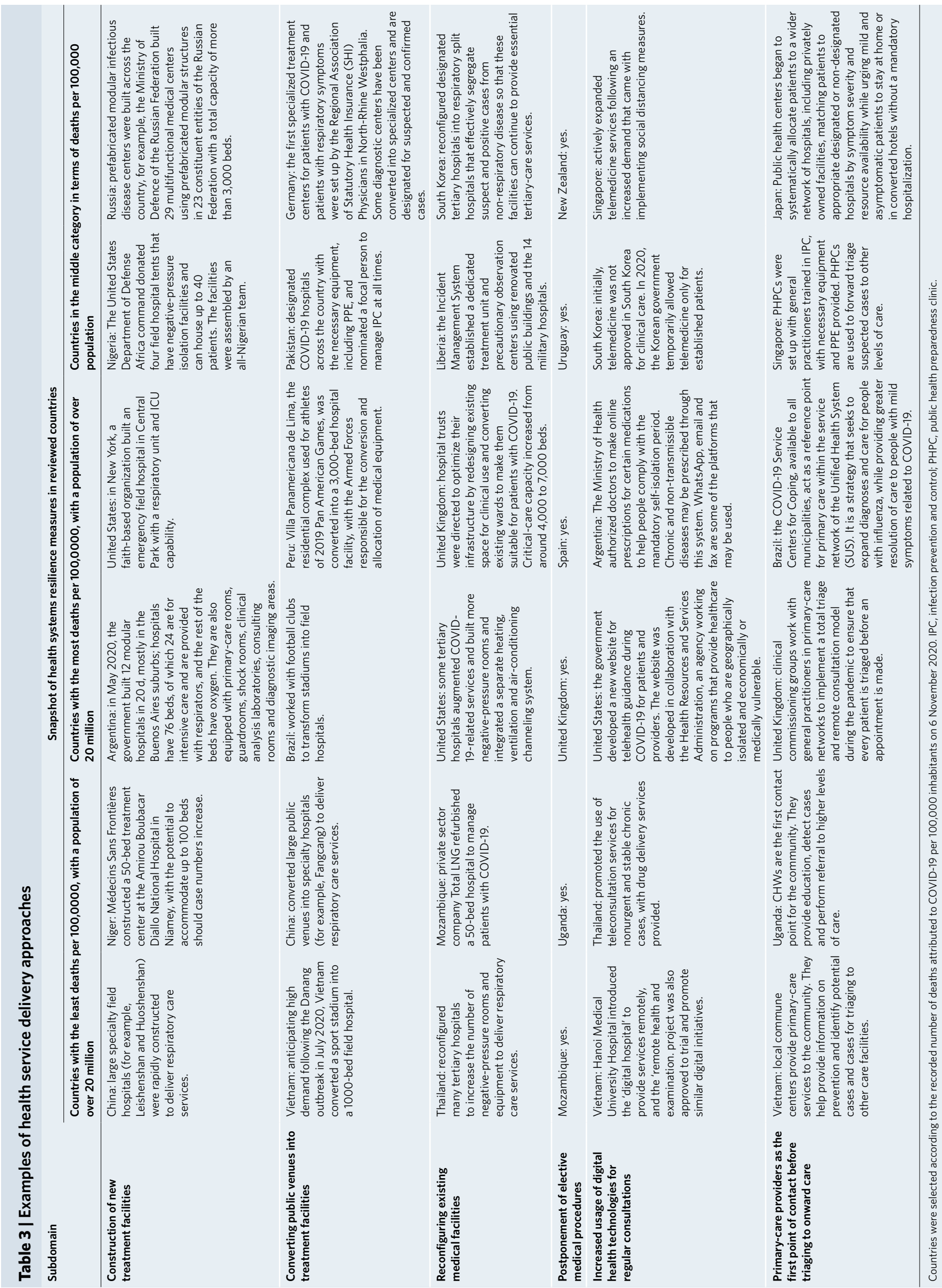




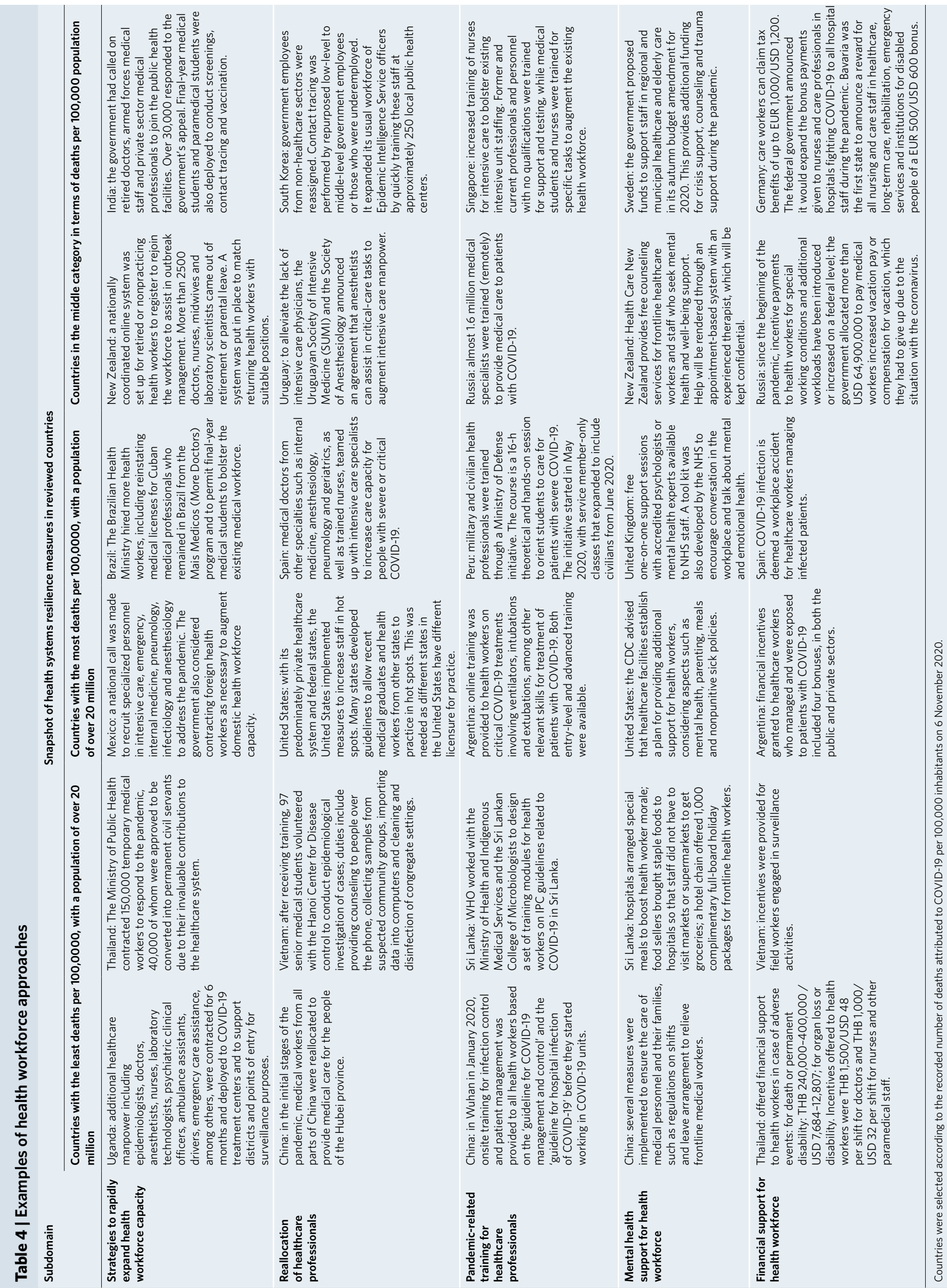




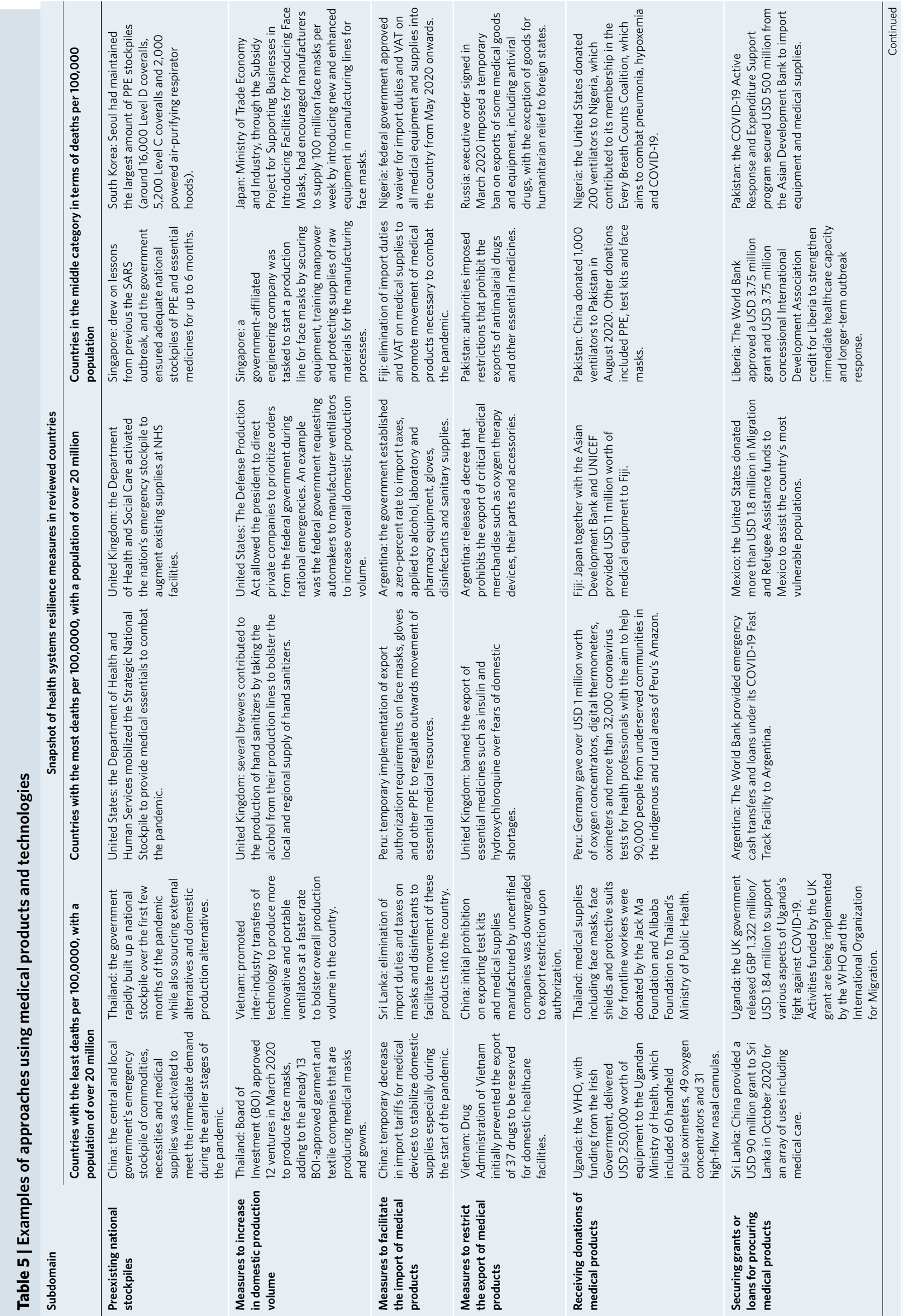




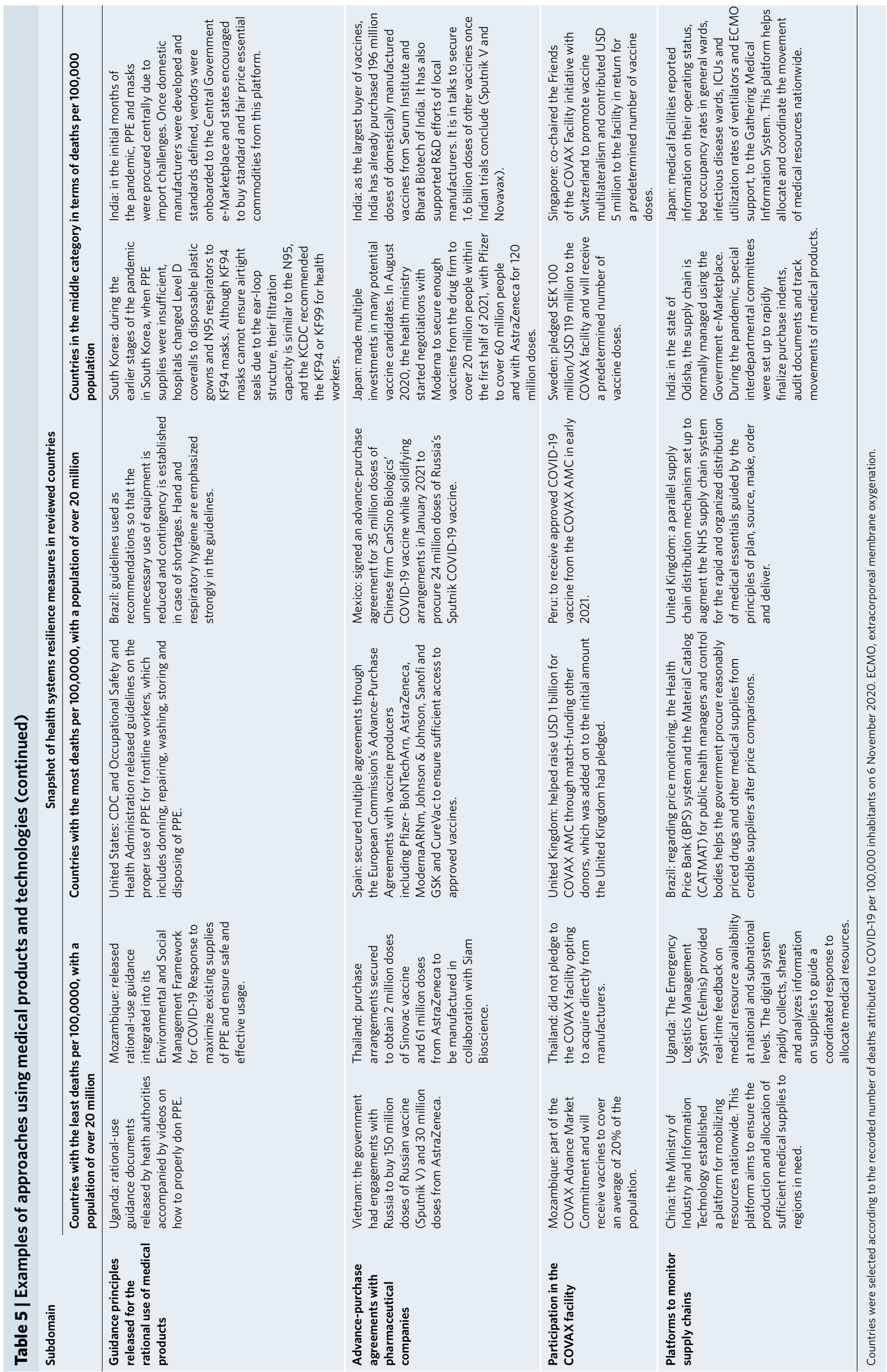




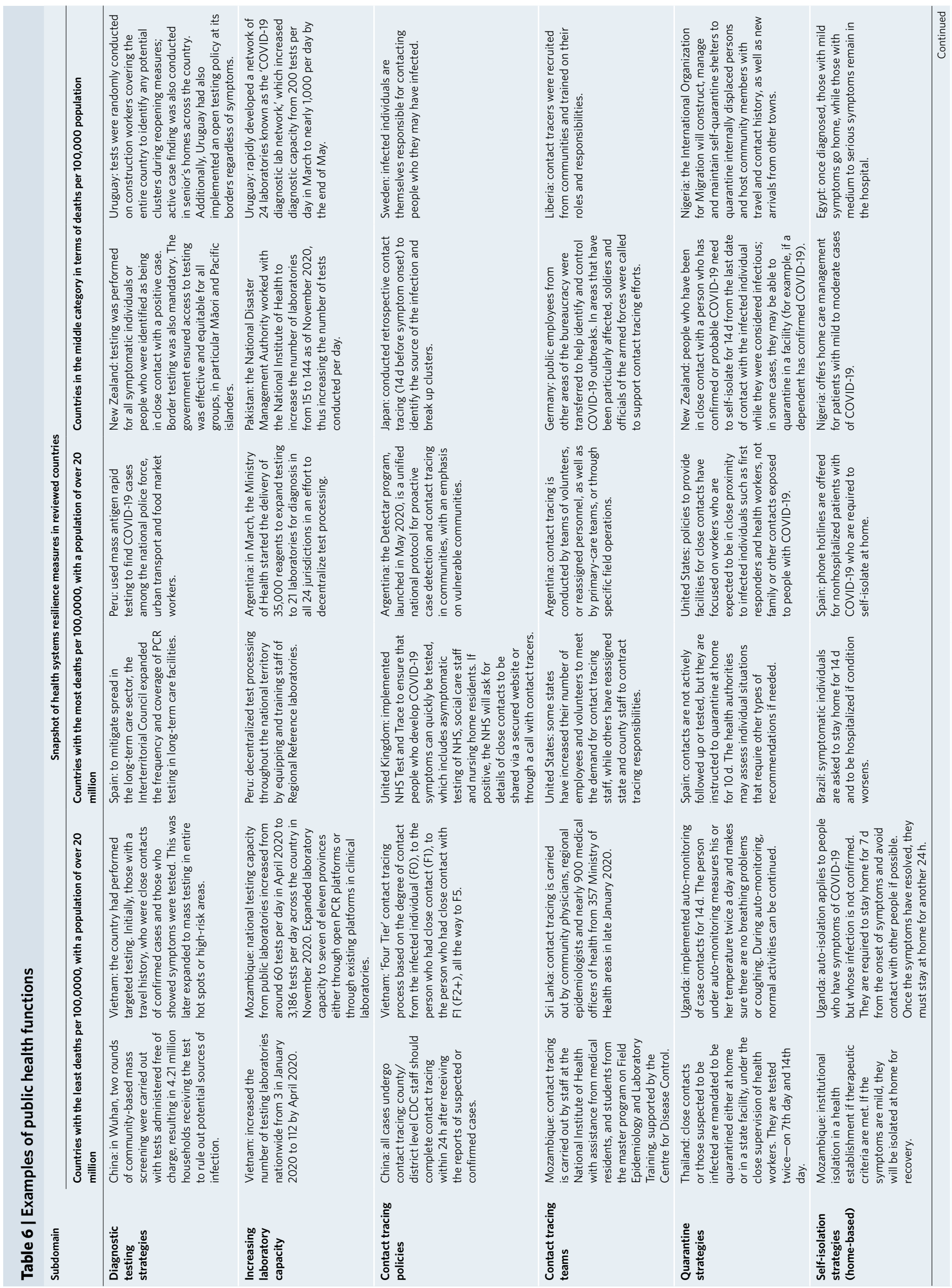




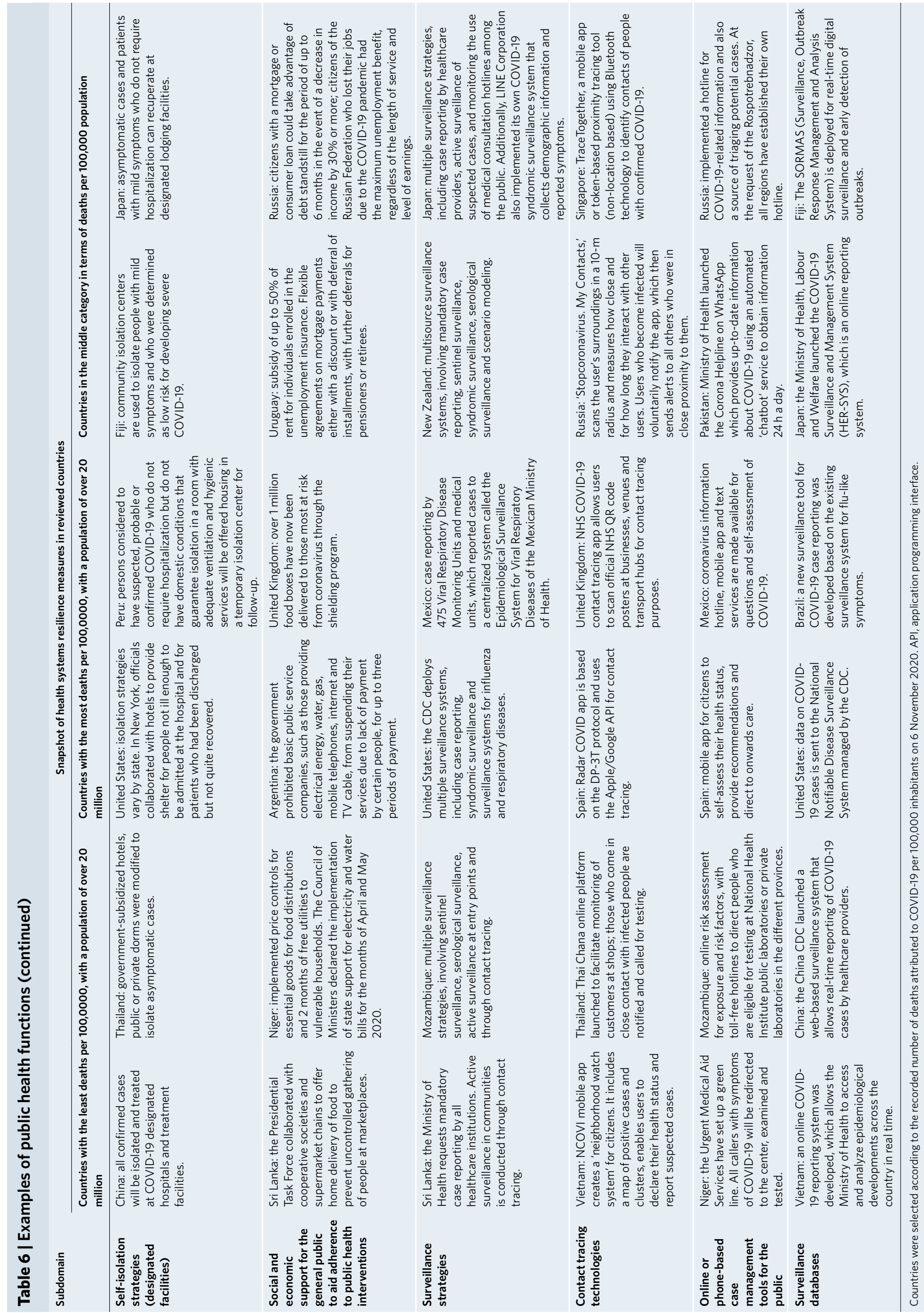


symptoms of COVID-19 to onward care ${ }^{24}$. Additionally, some countries complemented digital technology with proactive deployment of existing and new community health resources ${ }^{25}$. Community-based approaches developed with deep knowledge of local contexts are crucial to pandemic response and health systems resilience, particularly given the disproportionate impact of the pandemic on vulnerable groups ${ }^{15,26}$. While outside the reach of the health system in many countries, there have been extensive outbreaks in long-term care homes with devastating impacts on the health and well-being of high-risk older adults, long-term care patients and their fami$\operatorname{lies}^{27}$. In response, most countries reviewed prioritized long-term care facilities and older adults for testing, surveillance and vaccine distribution, although often not until there had been high rates of mortality in these settings.

Health workforce. Resilient health systems manage crises by having an adequate, trained and willing workforce. Yet, in many countries, COVID-19 has spread quickly among health workers as they have been the most exposed to the virus, with data indicating that they have been disproportionately affected by the pandemic ${ }^{28}$. Health workforce challenges during COVID-19 include low staffing levels (particularly among nurses) and uneven geographical distribution, shortages of adequate personal protective equipment (PPE), limited testing capacity, insufficient training, social discrimination and attacks and poor mental health ${ }^{29}$.

As cases surged globally, most reviewed countries reallocated healthcare professionals, including primary-care workers, to emergency care wards, intensive care units (ICUs) and diagnosis and surveillance activities. Several recruitment strategies were implemented to increase the healthcare workforce. Retired, student or nonpracticing medical and paramedical professionals were asked to volunteer for healthcare tasks. For example, medical and nursing students were recruited and allowed to perform supervised work in different COVID-19 response capacities in countries such as Germany, Russia, Spain, the United Kingdom and Vietnam. Given these new roles or expanded job scopes, there was an immediate need for rapid and high-quality pandemic-related training of frontline healthcare workers, which was accomplished through virtual training courses in many countries.

Further measures were taken by countries to maintain, protect and support their healthcare workers in light of the physical and psychological strain of the pandemic. In some countries (for example, Japan, Mozambique, Singapore and South Korea), healthcare professionals were supported by measures such as organization of shifts to avoid extended hours without rest, leaves from duty for mental and physical recovery, accommodation near their workplaces to protect their families, and childcare. Additionally, most countries reported giving some form of financial support to their health workers, such as monetary incentives, bonuses, insurance, tax benefits, overtime pay, meal allowances, classification of their infections as an occupational disease or injury and declaring cause of death as work related. Several countries reported making psychological support available for health workers, such as counseling or trauma support, to maintain well-being and morale. Frontline staff and their families were especially vulnerable and were targeted for psychological interventions. Moreover, some countries launched social media campaigns that encourage people to show their pride, admiration and gratitude for healthcare workers to promote solidarity.

Medical products and technologies. High-quality prevention, diagnosis and management of COVID-19 require the ongoing development, production and sustained distribution of mass quantities of medical products and technologies. However, overreliance on a few countries for production, competition among countries and supply chain disruptions have caused global supply shortages. Some countries reviewed had national or regional stockpiles of PPE, including masks, gloves, face shields and gowns, which were used as a buffer while awaiting imported supplies or scale up of domestic production. Singapore, for example, drew from experience responding to SARS and preserved a national stockpile of medical products for up to 6 months ${ }^{30}$. To replenish stockpiles in Japan, medical product manufacturers were urged to boost production output, resulting in the tripling of production volume as factories operated $24 \mathrm{~h}$ a day ${ }^{31}$.

Governments also worked beyond the typical health sectors and developed guidelines and specifications for non-health sectors to supplement the existing medical product manufacturing lines. In India, automotive manufacturers were repurposed to produce low-cost ventilators and $\mathrm{PPE}^{32}$. Beyond industry, communities in some countries mobilized to bolster medical product supplies. Several countries had also relied on purchasing consortia, like the UN COVID-19 Supply Chain System and Africa Medical Supplies Platform, donations from development partners or grants and loans from other countries and international financial entities (that is, The World Bank and Asian Development Bank) to secure medical supplies $^{33-37}$. Vaccine procurement is also an essential part of the medical supply chain, and countries have either made advance-purchase agreements or participated in the COVAX facility to ensure prompt access to the vaccine for their populations, although concerns remain about equitable access to vaccines in short supply.

In response to increased demand driven by widespread community transmission, countries enacted laws to prevent hoarding and exploitative pricing, as well as policies prohibiting export of medical supplies, while relaxing import licensing requirements and tariffs ${ }^{38}$. Within health facilities, measures including rational-use guidelines, per WHO recommendations, and postponement of nonemergency medical procedures helped stretch existing medical inventories ${ }^{39}$. Although supply chains began to stabilize during 2020, many points of care globally faced uncertain stocks and reports of counterfeit medical essentials highlighting the need for secure supply pipelines. Platforms that assist in monitoring logistics networks are integral in ensuring a steady and rapid flow of medical products and technologies, promoting transparency and ensuring better management of supply chains.

Public health functions. Public health interventions embedded within communities, such as testing, contact tracing, quarantine or self-isolation, and surveillance are crucial functions to break chains of transmission ${ }^{40}$. However, in many health systems, public health and health service delivery systems are siloed: their coordination is limited or ineffective, and they have separate referral processes and reporting systems, all of which serve to undermine health systems resilience ${ }^{41}$. Testing and contact tracing are a case in point that clearly illustrates why public health and health systems must act together in a coordinated manner.

Diagnostic tests to identify whether a person is, or has been, infected are foundational to infectious disease responses to pinpoint locations of spread, and provide care and treatment if it exists. Testing strategies are broadly classified as passive or proactive. Many of the countries reviewed relied on passive testing strategies, where symptomatic individuals self-present to a healthcare facility for testing after meeting certain criteria. However, some countries adopted proactive testing strategies characterized by programs tailored to the unique needs of specific populations as an important tool toward breaking chains of transmission and offering a clearer epidemiological picture ${ }^{42,43}$. Additionally, many countries rapidly decentralized testing capacity by strengthening or developing new laboratory networks.

Proactive testing must be accompanied by comprehensive contact tracing in partnership with communities. Contact tracing is the systematic process of following up with individuals who may have been exposed to COVID-1944. It can be characterized as either forward, aiming to find 'downstream' individuals who have been in 


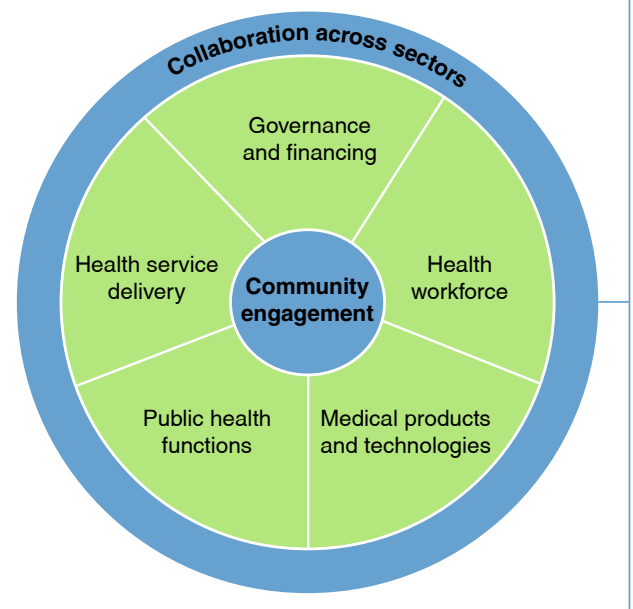

Activate comprehensive responses

- Multi-ministry task forces or committees and whole-of-government approach

- Training on COVID-19 including infection prevention and control for all health workers

- Advance purchase agreements and participation for novel medical products (e.g., participation in COVAX)

- Active surveillance, testing and contact tracing mechanisms supported by networks of laboratories

- Quarantine and isolation facilities, routine press briefings and regular updates on the situation

\section{Adapt health system capacity}

Public-private approaches that are cost-effective, accountable and transparent

- Financial and social supports for health workers

- Reallocation and recruitment of health workers, including retired or foreign-trained health workers and trainees

- Access and use of digital technologies

- Creation of temporary healthcare facilities or adaptation of existing civic facilities

- Postponing elective procedures

Preserve health system functions and resources

- Cost-effective procurement through purchasing consortia participation

- Rational-use guidelines to maximize and preserve medical resources

- Domestic research, development and production of PPE, test kits and other medical equipment

- Support CHWs so that public health can reach local populations

Support primary care for response activities and ongoing routine and acute care

- Engage the community in the planning of health services

Reduce vulnerability

- Financing mechanisms to provide relief for businesses, individuals and families

- Leverage the skills and knowledge of $\mathrm{CHWs}$ or other community leaders (e.g., youth groups and religious groups) to reach the general population and at-risk subpopulations

- Manufacturing and supply chain strengthening

- Proactive testing and contact tracing strategies

- Communication strategies that reach people where they are, e.g., social media, WhatsApp and public campaigns

Fig. 3 | Four resilience elements of highly effective country responses. The framework presented expands upon and applies the determinants of health systems resilience framework to identify four resilience elements characteristic of highly effective country responses to COVID-19.

contact with a person with COVID-19, or backwards, aiming to find an 'upstream' source of infection ${ }^{45}$. While most countries reviewed conducted forward contact tracing, Japan conducted backwards contact tracing measures aimed at identifying and 'busting' clusters by working with individuals to trace $14 \mathrm{~d}$ before symptom onset ${ }^{46}$. Contact tracing, particularly backwards tracing, is labor and time intensive and may be stigmatizing if not done with community engagement and consideration of at-risk and vulnerable groups ${ }^{47}$. The majority of countries reviewed introduced digital contact tracing tools. Even when fully operational, they may not be accessible, acceptable or feasible for use among those with limited access to, or concerns in using, adequate technology such as migrants, refugees or those experiencing homelessness, among others ${ }^{48}$.

Once cases and contacts are identified, self-isolation and quarantine measures are crucial to prevent onwards transmission and identify emergent cases. All countries reported on quarantine and isolation protocols. While necessary to outbreak management, unless done in coordination with communities, quarantine measures can have negative impacts on mental health and well-being, become a source of stigma and be deployed at the significant cost of human rights ${ }^{49}$. Some countries have implemented policies to provide social and economic assistance to those who must self-isolate or quarantine. Social supports range from services that ensure food and necessities during quarantine to dedicated quarantine or isolation facilities (for example, converted hotels, public facilities or purpose-built quarantine hospitals). Such self-isolation supports are recognized as integral to mitigating transmission, particularly among younger people and those working in high-exposure occupations, living in overcrowded housing or without a home ${ }^{50}$. However, to avoid negative unintended consequences, such facilities must be operationalized with a human rights focus ${ }^{51}$.

These efforts are enhanced by surveillance, including testing in areas or settings with outbreaks, to rapidly limit community circulation $^{52}$. Given the high transmissibility of severe acute respiratory syndrome coronavirus 2 , surveillance needs to be geographically comprehensive to provide accurate depictions of disease burden and epidemiology to prevent and mitigate community transmission ${ }^{53-55}$. As recommended by WHO guidelines, nearly all countries have adapted existing surveillance system infrastructure to collect information on COVID-19 cases $^{55}$. However, surveillance based on case reporting may underestimate the epidemiological characteristics of COVID-19, given that stigma or other barriers may limit healthcare seeking, particularly in vulnerable populations ${ }^{56,57}$. Therefore, New Zealand, Sweden and the United States have additionally deployed syndromic surveillance, which monitors cases that meet the clinical definition of COVID-19 without confirmation by testing. By implementing active surveillance approaches, countries have expanded surveillance coverage from healthcare settings into communities, such as through primary care, thereby strengthening epidemiological surveillance among vulnerable populations.

Timely sharing of case-based data between public health and healthcare sectors is key to early detection of outbreaks, identification of changes in epidemiological trends and planning of health services $^{58}$. This was facilitated by the use of innovative digital technology. For example, the China CDC launched a web-based infectious disease reporting system that allows real-time reporting of confirmed and suspected COVID-19 cases by healthcare providers $^{59,60}$. Similar real-time surveillance and information systems are also used in Fiji, India, Japan and Vietnam.

\section{Discussion}

Assessing health systems resilience is vital in helping policymakers plan for sustainable recovery and strengthen systems to better prepare and respond to current and future crises. Using an adapted and improved resilience framework, our review highlights many parallels in the measures implemented by countries in response to COVID-19. The similarities across countries with divergent health outcomes makes clear that there is no one silver bullet toward a resilient health system. Nevertheless, there are a number of characteristics of well-performing countries across the resilience 
determinants that stand out. These are summarized as four elements of resilience that are featured in highly effective country responses. These elements draw on the concept that resilient health systems are systems that: (1) activate comprehensive responses, which are responses that consider and address health and well-being as intertwined with social and economic considerations; (2) adapt capacity within and beyond the health system to meet the needs of communities; (3) preserve functions and resources within and beyond the health system to maintain pandemic-related and non-related routine and acute care; and (4) reduce vulnerability to catastrophic losses in communities, both in terms of health and well-being, as well as individual or household finances; all while continually learning, monitoring and adjusting in light of emerging evidence or the evolving epidemiological situation (Fig. 3).

High-performing countries. High-performing countries activated comprehensive responses across the determinants' domains, including through whole-of-government approaches and the creation of multi-ministry task forces, to ensure adequate translation of evidence into policy and practices that preserve health system capacity, while protecting public health and livelihoods. Specific measures taken include training health workers, bolstering public health functions (including offering designated isolation facilities, either for all or for those unable to safely self-isolate at home) and preparing for new technologies and medicines through purchase agreements, while also engaging communities through routine communications on the epidemiological situation and emergent policies.

These countries also learned from emerging evidence and adapted the capacity of their health system in response to the evolving epidemiological situation. This was achieved by increasing capacity in hospitals, through construction of makeshift hospitals or repurposing of existing health facilities or civic spaces. The health workforce in high-performing countries was expanded through reallocation and recruitment and supported through financial and social supports.

These countries took action to preserve health system functions and resources through purchasing consortia and rational-use guidelines to maximize available material resources such as PPE, as well as investing in domestic research, development and production of medical supplies, test kits and vaccines. Additionally, these countries protected health and well-being more broadly by ensuring health system functioning for non-COVID-19-related health services. High-performing countries supported primary care and CHWs to conduct COVID-19 screening, assessment and/or referral, while providing ongoing routine and acute care in communities.

High-performing countries also sought to reduce vulnerability across the resilience determinants by providing financial relief and social supports to complement proactive and robust testing and contact tracing in partnership with communities to ensure public health measures and safety net supports reached all groups.

Building resilient health systems. While some countries have demonstrated elements of resilience, as we highlight above, progress is limited in developing resilient health systems overall. Our review highlights six areas requiring urgent action to build resilient health systems globally.

First, COVID-19 responses provide a clear illustration of the importance of governance supported by scientific evidence and leadership willing to learn and adjust course for successful health systems that protect health and well-being. Enhancing resilience to future disease outbreaks requires longer-term work to create high-quality healthcare systems and build community trust. Our review emphasizes that governments are well advised to address COVID-19, and any future disease outbreak, through a whole-of-government approach that incorporates all sectors, engages relevant actors across all levels, including community and local authorities, and is based on strong and clear coordination that extends beyond early-stage emergency management ${ }^{61}$. Crucial to health systems resilience is that governance must consider the intersections of gender, racialization and human rights, and their impact on health and well-being before, during and after crises ${ }^{62-64}$. Urging governments to adopt such an approach, which COVID-19 has made clear is essential, is not a new proposal. Yet, our review highlights a lack of uniform appreciation or adoption of such an approach by countries.

Second, health systems need appropriate financing, not only to prepare for new pandemics, but also to ensure that at all times, all people have access to the health services they need, when and where they need them, without financial hardship, regardless of ability to pay $^{65}$. This is the foundation of UHC. While many countries have provided subsidized COVID-19 testing and treatment, more must be done to ensure people are not pushed into poverty due to out-of-pocket spending on health. Investing in UHC not only protects people from health threats but also mitigates the social and economic burdens that have characterized COVID-19. Countries will have to revisit the thresholds of health expenditure that they are willing to invest to build resilient health systems, promote population health and protect communities against financial risk.

Third, while country capacities varied, the pandemic has demonstrated a need to invest in improving both the quantity and quality of health workers to better prepare for and respond to future pandemics. Our review highlights that resilient health systems are those that not only invest in pandemic-related planning and training of health workers, but also ensure their physical, mental and economic protection in the workplace and beyond. Emphasis should also be placed on community mobilization where adequately trained and supported CHWs are equipped to play substantial roles in outbreak response and community engagement, much as they have played a crucial role in tuberculosis and HIV/AIDS response efforts globally for decades ${ }^{66}$.

Fourth, in terms of access to medicines and products, the pandemic has made visible, yet again, the clearly identified and thoroughly debated challenges to global supply chains for medicines and products. These challenges range from limited manufacturing capacities to financing to equity in access. The early experience of COVAX, with some high-income countries bypassing the initiative, has demonstrated the glaring limitations in the current system.

Fifth, health service delivery, including non-COVID-19-related health services, has been directly threatened, and often compromised, at all levels by the demands of the pandemic, even in traditionally high-performing health systems. Our review emphasizes that bolstering system capacity requires strong and well-funded primary care, with a skilled and protected workforce, to ensure that high-quality care is delivered in communities, with strengthened linkages to public health systems. Similarly, the long-term care sector, and care for older adults, must be prioritized and better integrated into health service delivery and public health functions. This must be underpinned by a renewed commitment to UHC to ensure high-quality care for all.

Finally, public health functions, such as testing and contact tracing, that are delivered in coordination with the health service system, are cornerstones for successful COVID-19 responses. These approaches often depend on innovative digital technologies, which bear their own challenges, including the potential to exacerbate inequalities and be the vehicle for human rights violations $s^{67-70}$. As such, future investments in these technologies requires a more holistic approach-one that engages communities, particularly the most vulnerable-that takes into account the potential risks and considers how health systems can minimize harms from their use ${ }^{71}$.

Importantly, our analysis of country responses points toward foundational debates on how we understand and think about resilient health systems. Health system resilience as a concept must 
expand beyond technical and biomedical knowledge and actions, to engage with the broader social, economic and political factors in society. Such comprehensive understanding of resilience requires a systems approach and should be guided by equity concerns, which include concerns for gender, human rights and racialization in health and healthcare ${ }^{12,72}$. Further, resilience cannot be achieved solely through unidirectional and top-down approaches by governments and other entities. Resilience requires community engagement as much as regulations and hospital capacity. Community engagement and its interlinkage with community resilience is fundamental to managing not only health threats but also other threats, such as climate and environmental change ${ }^{73}$. Importantly, health systems resilience requires countries worldwide to be open to exchange of knowledge and expertise from regions such as Asia and Africa, which have effectively mobilized CHWs and communities to extend the reach, capacity and quality of their health systems.

\section{Conclusion}

Our findings and recommendations are not new, and there have been prior incremental moves to expand what constitutes, influences and governs health and healthcare. COVID-19 demands dynamic systemic transformation. The pandemic has fundamentally challenged health systems and the communities they serve globally. The effect of a major shock represented by the pandemic is to manifest the points where the system is weakest, and to demonstrate the interdependencies of a range of health, social and economic structures. While the evidence of system failures has come at a huge cost in human and monetary terms, it has also pointed to what needs to change. With over 3 million global deaths and pervasive social and economic costs, the pandemic must serve as a call for transformation and investment toward resilience and people centeredness, beginning with health systems. COVID-19 provides a renewed prospect for solidarity, both within and between countries. It also serves as a reminder that health is more than healthcare and that a whole-of-government approach to health and well-being is needed to create healthy populations able to collectively prevent and respond to crises, leaving no one behind.

Received: 8 March 2021; Accepted: 29 April 2021; Published online: 17 May 2021

\section{References}

1. World Health Organization. WHO Coronavirus Disease (COVID-19) Dashboard. https://covid19.who.int (2021).

2. Glover, R. E. et al. A framework for identifying and mitigating the equity harms of COVID-19 policy interventions. J. Clin. Epidemiol. 128, 35-48 (2020).

3. Baker, M. G., Wilson, N. \& Blakely, T. Elimination could be the optimal response strategy for COVID-19 and other emerging pandemic diseases. BMJ 371, m4907 (2020).

4. Etienne, C. F. et al. COVID-19: transformative actions for more equitable, resilient, sustainable societies and health systems in the Americas. BMJ Glob. Health 5, e003509 (2020).

5. El Bcheraoui, C., Weishaar, H., Pozo-Martin, F. \& Hanefeld, J. Assessing COVID-19 through the lens of health systems' preparedness: time for a change. Global Health 16, 112 (2020).

6. Kruk, M. E., Myers, M., Varpilah, S. T. \& Dahn, B. T. What is a resilient health system? Lessons from Ebola. Lancet 385, 1910-1912 (2015).

7. United Nations System Chief Executives Board for Coordination (CEB). United Nations plan of action on disaster risk reduction for resilience. https://www.preventionweb.net/files/49076_unplanofaction.pdf (2016).

8. Nuzzo, B. et al. What makes health systems resilient against infectious disease outbreaks and natural hazards? Results from a scoping review. BMC Public Health 19, 1310 (2019).

9. Haldane, V., Ong, S. E., Chuah, F. L. \& Legido-Quigley, H. Health systems resilience: meaningful construct or catchphrase? Lancet 389, 1513 (2017).

10. Legido-Quigley, H. \& Asgari, N. Resilient and people-centred health systems: progress, challenges and future directions in Asia (World Health Organization, 2018).

11. Legido-Quigley, H. et al. Are high-performing health systems resilient against the COVID-19 epidemic? Lancet 395, 848-850 (2020).
12. Haldane, V. \& Morgan, G. T. From resilient to transilient health systems: the deep transformation of health systems in response to the COVID-19 pandemic. Health Policy Plan. https://doi.org/10.1093/heapol/czaal69 (2020).

13. World Health Organization. Monitoring the building blocks of health systems: a handbook of indicators and their measurement strategies (2010).

14. Barker, K. M. et al. Community engagement for health system resilience: evidence from Liberia's Ebola epidemic. Health Policy Plan. 35, 416-423 (2020).

15. Shadmi, E. et al. Health equity and COVID-19: global perspectives. Int. J. Equity Health 19, 1-16 (2020).

16. Monaghesh, E. \& Hajizadeh, A. The role of telehealth during COVID-19 outbreak: a systematic review based on current evidence. BMC Public Health 20, 1193 (2020)

17. Gilmore, B. et al. Community engagement for COVID-19 prevention and control: a rapid evidence synthesis. BMJ Glob. Health 5, e003188 (2020).

18. Bonell, C. et al. Harnessing behavioural science in public health campaigns to maintain 'social distancing' in response to the COVID-19 pandemic: key principles. J. Epidemiol. Community Health 74, 617-619 (2020).

19. Ballard, M. et al. Prioritising the role of community health workers in the COVID-19 response. BMJ Glob. Health 5, e002550 (2020).

20. Nigeria Centre for Disease Control. NCDC and UNICEF launch Chatbot to combat COVID-19 misinformation in Nigeria. https://ncdc.gov.ng/news/ 272/ncdc-and-unicef-launch-chatbot-to-combat-covid-19-misinformationin-nigeria (2020).

21. Luo, H., Liu, J., Li, C., Chen, K. \& Zhang, M. Ultra-rapid delivery of specialty field hospitals to combat COVID-19: lessons learned from the Leishenshan Hospital project in Wuhan. Autom. Constr. 119, 103345 (2020).

22. Her, M. Repurposing and reshaping of hospitals during the COVID-19 outbreak in South Korea. One Health 10, 100137 (2020).

23. World Health Organization. Coronavirus disease (COVID-19) technical guidance: patient management. http://www.who.int/emergencies/diseases/ novel-coronavirus-2019/technical-guidance/patient-management (2020).

24. Ministry of Health New Zealand. COVID-19: advice for all health professionals. https://www.health.govt.nz/our-work/diseases-andconditions/covid-19-novel-coronavirus/covid-19-information-healthprofessionals/covid-19-advice-all-health-professionals (2020).

25. Haldane, V. et al. National primary care responses to COVID-19: a rapid review of the literature. BMJ Open 10, e041622 (2020).

26. World Health Organization Western Pacific. COVID-19: vulnerable and high risk groups. http://www.who.int/westernpacific/emergencies/ covid-19/information/high-risk-groups (2020).

27. McMichael, T. M. et al. Epidemiology of COVID-19 in a long-term care facility in King County, Washington. N. Engl. J. Med. 382, 2005-2011 (2020).

28. Bandyopadhyay, S. et al. Infection and mortality of healthcare workers worldwide from COVID-19: a systematic review. BMJ Glob. Health 5, e003097(2020)

29. World Health Organization. Health workforce policy and management in the context of the COVID-19 pandemic response (2020).

30. Chua, A. Q. et al. Health system resilience in managing the COVID-19 pandemic: lessons from Singapore. BMJ Glob. Health 5, e003317 (2020).

31. Ministry of Economy, T. and I. Current status of production and supply of face masks, antiseptics and toilet paper. https://www.meti.go.jp/english/ covid-19/mask.html (2020).

32. Mukherjee, S. Maruti Suzuki to help produce ventilators, masks and protective equipment to fight against COVID-19 (2020).

33. World Bank. 100 Countries Get Support in Response to COVID-19 (2020).

34. World Bank. World Bank group's operational response to COVID-19 (coronavirus)-projects list (2020).

35. World Health Organization. COVID-19 supply chain system: requesting and receiving supplies. http://www.who.int/publications/m/item/covid-19. supply-chain-system-requesting-and-receiving-supplies (2020).

36. ABOUT US. Africa Medical Supplies Platform. https://amsp.africa/about-us/

37. World Health Organization. UK Gives $£ 1.3$ million to fight COVID-19 in Uganda. https://www.afro.who.int/news/uk-gives-ps13-million-fightcovid-19-uganda (2020).

38. International Trade Center. COVID-19 temporary trade measures. https:// www.macmap.org/covid19 (2020).

39. World Health Organization. Rational use of personal protective equipment for coronavirus disease (COVID-19) and considerations during severe shortages. https://www.who.int/publications/i/item/rational-use-ofpersonal-protective-equipment-for-coronavirus-disease-(covid-19)-andconsiderations-during-severe-shortages (2020).

40. Koo, D., Felix, K., Dankwa-Mullan, I., Miller, T. \& Waalen, J. A call for action on primary care and public health integration. Am. J. Public Health 102, S307-S309 (2012).

41. Nishtar, S. The mixed health systems syndrome. Bull. World Health Organ. 88, 74-75 (2010).

42. Veillard, J., Campbell, J., Mohpal, A. \& Evans, T. Testing, testing, testing: an essential strategy for public health, vaccine deployment and economic 
reactivation during COVID-19. https://blogs.worldbank.org/latinamerica/ testing-testing-testing-essential-strategy-public-health-vaccine-deploymentand/ (World Bank Blogs, 2020).

43. The World Bank. Population-level, national testing strategies for COVID-19: Latin America \& the Caribbean. https://www.worldbank.org/en/region/ lac/brief/population-level-national-testing-strategies-for-covid-19-latinamerica-and-the-caribbean (2020).

44. World Health Organization. Contact tracing in the context of COVID-19. https://www.who.int/publications/i/item/contact-tracing-in-the-contextof-covid-19 (2020)

45. Endo, A. et al. Implication of backward contact tracing in the presence of overdispersed transmission in COVID-19 outbreaks. Wellcome Open Res. 5, 239 (2020).

46. Lewis, D. Why many countries failed at COVID contact-tracing-but some got it right. Nature 588, 384-387 (2020).

47. Megnin-Viggars, O., Carter, P., Melendez-Torres, G. J., Weston, D. \& Rubin, G. J. Facilitators and barriers to engagement with contact tracing during infectious disease outbreaks: a rapid review of the evidence. PLOS ONE 15, e0241473 (2020)

48. Klenk, M. \& Duijf, H. Ethics of digital contact tracing and COVID-19: who is (not) free to go? Ethics Inf. Technol. https://doi.org/10.1007/s10676-02009544-0 (2020).

49. Brooks, S. K. et al. The psychological impact of quarantine and how to reduce it: rapid review of the evidence. Lancet 395, 912-920 (2020).

50. Cevik, M., Baral, S., Crozier, A. \& Cassell, J. Support for self-isolation is critical in COVID-19 response. BMJ 372, 224 (2021).

51. Social Science in Humanitarian Action. Key considerations: quarantine in the context of COVID-19. https://opendocs.ids.ac.uk/opendocs/bitstream/ handle/20.500.12413/15133/SSHAP\%20COVID-19\%20Key\%20 Considerations\%20Quarantine.pdf? sequence=24\&isAllowed $=y$ (2020).

52. Pung, R. et al. Investigation of three clusters of COVID-19 in Singapore: implications for surveillance and response measures. Lancet 395 , 1039-1046 (2020)

53. Hao, X. et al. Reconstruction of the full transmission dynamics of COVID-19 in Wuhan. Nature 584, 420-424 (2020).

54. Hu, B., Guo, H., Zhou, P. \& Shi, Z.-L. Characteristics of SARS-CoV-2 and COVID-19. Nat. Rev. Microbiol. 19, 141-154 (2021).

55. World Health Organization. Public health surveillance for COVID-19: interim guidance (2020).

56. Chiolero, A., Santschi, V. \& Paccaud, F. Public health surveillance with electronic medical records: at risk of surveillance bias and overdiagnosis. Eur. J. Public Health 23, 350-351 (2013).

57. Alwan, N. A. Surveillance is underestimating the burden of the COVID-19 pandemic. Lancet 396, e24 (2020).

58. Jajosky, R. A. \& Groseclose, S. L. Evaluation of reporting timeliness of public health surveillance systems for infectious diseases. BMC Public Health 4 , 1-9 (2004).

59. Khan, M. S., Wu, S., Wang, X. \& Coker, R. Optimising routine surveillance systems for informing tuberculosis control policies in China. Health Policy Plan. 32, i12-i14 (2017).

60. China CDC. The launch of infectious disease reporting system for COVID-19 (2020).

61. WHO regional office for Europe. Strengthening the health system response to COVID-19: recommendations for the WHO European region (2020).

62. Davies, S. E. \& Bennett, B. A gendered human rights analysis of Ebola and Zika: locating gender in global health emergencies; a gendered human rights analysis of Ebola and Zika: locating gender in global health emergencies. Int. Aff. 92, 1041-1060 (2016).

63. Harman, S. Ebola, gender and conspicuously invisible women in global health governance. Third World Q 37, 524-541 (2016).

64. Wenham, C., Smith, J. \& Morgan, R., Gender and COVID-19 Working Group. COVID-19: the gendered impacts of the outbreak. Lancet 395, 846-848 (2020).
65. World Health Organization. Universal health coverage (UHC). https://www.who.int/news-room/fact-sheets/detail/universal-healthcoverage-(uhc) (2019).

66. Bhutta, Z. A., Lassi, Z., Pariyo, G. \& Huicho, L. Global Experience of Community Health Workers for Delivery of Health Related Millennium Development Goals: a systematic review, country case studies, and recommendations for integration into national health systems. https://www. who.int/workforcealliance/knowledge/publications/alliance/Global_CHW_ web.pdf (2010).

67. Keshet, Y. Fear of panoptic surveillance: using digital technology to control the COVID-19 epidemic. Isr. J. Health Policy Res. 9, 67 (2020).

68. Enter the cyborgs: health and human rights in the digital age. Health Hum. Rights https://www.hhrjournal.org/2020/12/editorial-enter-the-cyborgshealth-and-human-rights-in-the-digital-age/ (2020).

69. Technology, health, and human rights: a cautionary tale for the post-pandemic world. Health Hum. Rights https://www.hhrjournal.org/ 2020/12/viewpoint-technology-health-and-human-rights-a-cautionarytale-for-the-post-pandemic-world/ (2020).

70. Analyzing the human rights impact of increased digital public health surveillance during the COVID-19 crisis. Health Hum. Rights https://www. hhrjournal.org/2020/12/analyzing-the-human-rights-impact-of-increaseddigital-public-health-surveillance-during-the-covid-19-crisis/ (2020).

71. Lal, A., Erondu, N. A., Heymann, D. L., Gitahi, G. \& Yates, R. Fragmented health systems in COVID-19: rectifying the misalignment between global health security and universal health coverage. Lancet 397, 61-67 (2021)

72. Baum, F. et al. Explaining COVID-19 performance: what factors might predict national responses? BMJ 372, n91 (2021).

73. Ebi, K. L. \& Semenza, J. C. Community-based adaptation to the health impacts of climate change. Am. J. Prev. Med. 35, 501-507 (2008).

\section{Acknowledgements}

Data for this review were collected under the auspices of the Independent Panel for Pandemic Preparedness and Response. The analysis of this paper is separate from the Independent Panel's Final Report and has been facilitated by the Independent Panel Secretariat. The Secretariat of the Independent Panel for Pandemic Preparedness and Response is independent and impartial. The views expressed in this work are solely that of the authors and do not represent the views of the Independent Panel for Pandemic Preparedness and Response.

\section{Author contributions}

V.H., C.D.F., S.A., A.-S.J. and H.L.-Q. conceived and designed this Review. V.H., C.D.F., S.A., A.-S.J., M.M.J.T., S.W., A.C., M.V., P.S., S.M.T. and H.L.-Q. collected the data. V.H., C.D.F., S.A., A.-S.J., M.M.J.T., S.W., S.S. and H.L.-Q. analyzed the data and drafted the manuscript with input from all authors. All authors contributed to revision of the manuscript.

\section{Competing interests}

All authors declare no competing interests.

\section{Additional information}

Correspondence should be addressed to H.L.-Q.

Peer review information Nature Medicine thanks Ole F. Norheim and Margaret Kruk for their contribution to the peer review of this work. Joao Monteiro was the primary editor on this article and managed its editorial process and peer review in collaboration with the rest of the editorial team.

Reprints and permissions information is available at www.nature.com/reprints.

Publisher's note Springer Nature remains neutral with regard to jurisdictional claims in published maps and institutional affiliations.

(C) Springer Nature America, Inc. 2021 